\title{
DE CÓMO LAURA GARCÍA PERDIÓ SU ACENTO ${ }^{1}$ María López Ponz \\ Universidad de Salamanca
}

\begin{abstract}
The aim of this paper is to analyze how Julia Alvarez constructs the character of the mother, Laura García de la Torre, through the latter's use of language in How the Garcia Girls Lost Their Accents. Given that language is one of the main signs of identity, the way in which Laura expresses herself reveals her attitude towards American society, as well as her position within that culture. We would expect, therefore, to find these features in the translation. However, as we will see, neither of the Spanish versions deploys a systematic stance on this problem.
\end{abstract}

KEY WORDS: How the Garcia Girls Lost Their Accents, language, identity, translation, hybridization.

\section{RESUMEN}

El objetivo de este artículo es analizar cómo Julia Álvarez construye el personaje de la madre, Laura García de la Torre, a través de su forma de utilizar la lengua en How the Garcia Girls Lost Their Accents. Dado que el lenguaje es uno de los signos más claros de la identidad, la manera de expresarse de Laura revela una actitud frente a la sociedad estadounidense, así como una situación muy determinada dentro de esa cultura. Sería de esperar, por lo tanto, que estas características se hicieran patentes en la traducción, pero, como veremos, ninguna de las dos versiones existentes en español adopta una postura sistemática respecto a este problema.

PALABRAS CLAVE: How the Garcia Girls Lost Their Accents, lenguaje, identidad, traducción, hibridación.

\footnotetext{
${ }^{1}$ Este artículo se enmarca dentro de la actividad del Proyecto de Investigación de Excelencia de la Junta de Castilla y León GR277 "Inmigración y políticas de traducción: nuevos retos de la mediación intercultural en el siglo XXI”.
} 


\section{INTRODUCCIÓN}

Existen diversas maneras de caracterizar a un personaje en una obra literaria: hay autores que optan por describirlo y otros que lo definen por comparación con el resto de protagonistas; también hay quienes dejan que el lector vaya descubriendo su personalidad mediante sus actos y quienes prefieren no dar demasiada información para así retar a la imaginación de la audiencia. En How the García Girls Lost Their Accents, Julia Álvarez emplea varios de estos métodos para revelar la personalidad de los distintos personajes, pero reserva uno más original para la madre, Laura García de la Torre, quien es caracterizada mediante su forma de utilizar la lengua. El modo en que hacemos nuestro un idioma dice mucho acerca de nosotros, y Álvarez utiliza este recurso para arrojar luces y sombras sobre un personaje que, como veremos, es esencial en el relato.

Es evidente desde el momento en que leemos el título de la novela que el lenguaje va a desempeñar un rol fundamental en ella, algo común en las obras escritas por escritoras híbridas. En este caso, Álvarez utiliza la inmigración a Estados Unidos de la familia García para plantear una historia sobre la búsqueda de la identidad y la adaptación a un nuevo contexto en la que las palabras, los errores lingüísticos, las interferencias entre el inglés y el español, e incluso los silencios, tienen significado más allá de lo que parecen.

\section{HOW THE GARCÍA GIRLS LOST THEIR ACCENTS}

Julia Álvarez, escritora estadounidense de origen dominicano, publicó How the Garcia Girls Lost Their Accents en 1991 en Algonquin Books. A pesar de ser su primera novela, esta obra, según afirmó Jonathan Bing en Publishers Weekly, "made a resounding splash on the literary scene". El libro trata el paso a la adultez de las hermanas García-De la Torre. Carla, Sandra, Yolanda y Sofía viven en la República Dominicana en el marco de una familia acomodada; su padre, Carlos, médico de profesión, y su madre, Laura, proceden, como se nos cuenta en un capítulo del libro, de los Conquistadores, los primeros españoles en llegar a América y esto, unido a la fortuna familiar, les concede un cierto estatus social. Sin embargo, se ven obligados a huir del país debido a la participación del padre en el golpe de estado contra la dictadura de Rafael Leónidas Trujillo. La familia comienza una nueva vida en Nueva York, y el cambio resulta radical, no sólo por las diferencias geográficas, culturales y lingüísticas, sino también porque su estatus social y económico pasa a ser justo el contrario. 
Todos los miembros de la familia tienen que acomodarse a la nueva situación, aunque sin duda representa un conflicto mayor para las cuatro hijas, quienes viven su adolescencia y madurez en medio de dos culturas, la estadounidense y la dominicana, que tiran de ellas en sentidos contrarios. La novela, en buena parte autobiográfica, es fundamentalmente una Bildungsroman en la que a los conflictos típicos de este tipo de obras se suman otros derivados tanto del sentimiento de desplazamiento que sufren las hermanas al tener que forjar su identidad en un nuevo país, desarraigadas de su cultura de origen, pero profundamente influenciadas por ella, como de los problemas de género que surgen de la oposición entre la sumisión que su familia espera de ellas como mujeres y la libertad que la sociedad estadounidense les ofrece. Narrada en orden cronológico inverso, la historia comienza en 1989, cuando las cuatro hermanas ya son mujeres adultas, y termina en 1956, poco antes de emigrar, cuando aún son niñas y viven en la República Dominicana.

La sensación de habitar un espacio fronterizo, de estar entre dos culturas, y el consecuente mestizaje es un tema fundamental a lo largo de la novela, algo común en las obras de escritoras latinas e híbridas en general. Esta hibridación se hace especialmente presente en el lenguaje, que, como veremos luego, toma elementos de ambos idiomas para crear un medio de expresión que refleje la identidad de sus hablantes.

Si bien suelen ser las cuatro hermanas, especialmente Yolanda, las que acaparan la atención de los artículos escritos en torno a la novela, mi intención aquí es centrarme en el personaje de la madre y en cómo Álvarez apenas nos proporciona varias pinceladas sobre su personalidad y deja, en cambio, que sea su forma de utilizar el lenguaje la que nos ayude a vislumbrar su identidad. Es precisamente el análisis de la manera de traducir este rasgo característico al español lo que ocupa mi interés en este artículo, ya que su importante función como elemento definitorio en el texto original debería, a priori, mantenerse en la traducción.

No es mucha la información que se nos proporciona acerca de Laura; sabemos que proviene de una familia muy rica, privilegiada e influyente en la República Dominicana y que, por lo tanto, está acostumbrada a un estatus social y económico alto. También se nos dice que ha estudiado en los Estados Unidos y es capaz de hablar inglés, algo que, como veremos, será fundamental en el desarrollo de la historia. Es obvio que ha sido educada según lo que se espera en su cultura de origen de una mujer de su posición, es decir, que sea una señora de su casa y críe a sus hijas. Tener que huir de su país supone un cambio radical en sus expectativas y su forma de vida; sin embargo, aunque esta situación a menudo le resulte frustrante, pronto nos damos cuenta de que Laura es una mujer fuerte que, por encima de todo, ama profundamente a su marido y a sus hijas. La inmersión en la cultura estadounidense no sólo cambia 
su plan de vida, sino que además la lleva a replantearse su identidad y su lugar en el mundo que la rodea.

\section{EL LENGUAJE COMO SÍMBOLO DE LA INDEPENDENCIA FEMENINA}

Ya hemos comentado que uno de los temas de la novela es el conflicto de género; por desgracia, el machismo es una característica común en la cultura latina y, por lo tanto, una cuestión recurrente en las obras escritas por autoras hispanas. En estas sociedades, a las mujeres no se les suele permitir el acceso a la esfera pública, porque se considera que su lugar está en casa, cuidando de la familia; como consecuencia, en el caso de emigrar, puede ocurrir que nunca lleguen a aprender bien el idioma del nuevo país. Obviamente, esto conlleva una situación de aislamiento que puede ser muy perjudicial para su salud, ya que no dependen de ellas mismas para comunicarse en la sociedad de acogida. Solicitar ayuda en caso de maltrato, acceder a un trabajo bien remunerado, conseguir una formación superior o, simplemente, salir solas a la calle se convierten en tareas casi imposibles. Al no poder "traducirse" a la nueva lengua, estas mujeres se encuentran totalmente atadas a sus familias:

The failure to be translated becomes cruelly confining and the women find themselves dependent on either bilingual males or their bilingual children to provide them with lifelines to the outside world. Hence the right to exercise autonomous forms of translation (the immigrant woman herself in control of the translation situation) as opposed to heteronymous forms (others controlling the translation exchange) is seen as a crucial element in the emancipation of immigrant women and an important factor in their social and psychological well-being (Cronin, 2006: 53-4).

Sin embargo, puede ocurrir justo lo contrario, especialmente con las niñas, porque al acceder a la educación en el nuevo país, también acceden a nuevos modelos de comportamiento femenino. En estos casos, invaden el espacio que sus culturas reservan tradicionalmente a los hombres y, entonces, aparece el conflicto entre lo que sus familias esperan que sean y lo que ellas quieren ser o son. A pesar del amor que puedan sentir por sus raíces, se rebelan contra el orden patriarcal que les reserva una posición de inferioridad respecto a los hombres.

En cualquiera de los dos casos, resulta obvio que el lenguaje es un elemento fundamental en nuestras relaciones con el medio que nos rodea. Es una herramienta indispensable para la vida en sociedad y un instrumento para expresar nuestra identidad, como veremos en el siguiente epígrafe, pero también puede ser un referente poderoso, porque "aquel que maneja la 
información, los significados, adquiere poder" (Esquivel, 2007 [2005]: 80). Un buen manejo de la lengua puede convertirse en la mejor de las armas.

En How the Garcia Girls Lost Their Accents, el lenguaje tiene un papel fundamental, algo que observamos desde el principio, puesto que ya el título hace clara referencia a este tema. En el caso de las hijas el aprendizaje y manejo del inglés va unido a su adaptación a la cultura estadounidense. En ocasiones veremos que funciona como elemento de subversión frente a sus padres y a su actitud conservadora y machista, y, por lo tanto, Álvarez utiliza este aprendizaje, esta evolución en el uso del idioma, como un marcador de la separación cultural cada vez mayor entre padres e hijas. El dominio del lenguaje es, pues, un signo de independencia y emancipación femenina, un arma para deshacerse de rígidas convenciones sociales, una forma de invadir la esfera pública, vetada a las mujeres en la sociedad dominicana. ¿Y qué ocurre en el caso de la madre? Desde el principio vemos que, a pesar de sus frecuentes calcos sintácticos, Laura habla inglés mejor que su marido, pero no sabremos la razón hasta casi el final del libro cuando se nos cuenta la llegada de la familia a Estados Unidos y una de las hijas desvela el misterio: "Mami was the leader now that they lived in the States. She had gone to school in the States. She spoke English without a heavy accent" (Álvarez, 1991: 176). Es decir, Álvarez subvierte el patrón más común de familia emigrante al hacer que Laura domine la lengua de llegada y, por lo tanto, la situación, hasta cierto punto. No depende de su marido, sino al revés, es ella la que la que ejerce una forma autónoma de traducción y, de hecho, su adaptación a la sociedad estadounidense es mayor que la de Carlos. El hecho de que la novela esté escrita en orden cronológico inverso plantea una curiosa situación al lector, ya que no observa la evolución de Laura, sino su involución.

En mi opinión, la correspondencia entre el dominio de la lengua, la adaptación a la nueva cultura y las relaciones de las hijas con cada progenitor no es casual. Las cuatro hermanas adoran a su padre, pero no pueden comunicarse con él, no hablan el mismo idioma, las premisas desde las que parte su concepción del mundo son tan distintas que no son capaces de entenderse. Por ejemplo, cuando alguna de las hijas tiene un problema de salud, es Laura la que habla con el médico, la que trata de comprender y explicar la situación, mientras que Carlos se limita a callar y a observar confuso lo que ocurre, no es capaz de encontrar las palabras para desentrañar y traducir la realidad que viven sus hijas, porque no habita el mismo universo, ni cultural ni lingüístico.

En cambio, desde el comienzo de la novela (desde el final de la historia, podríamos decir) vemos que, a pesar de las evidentes diferencias generacionales, culturales y sociales, Laura sí se comunica con sus hijas. Ella es consciente de que el cambio de contexto a consecuencia de la emigración conlleva una serie de alteraciones, una evolución en la manera de ver el mundo. Ambas actitudes, la de Carlos y la de Laura, se hacen patentes en el capítulo 
"Daughter of Invention", cuando Yolanda tiene que escribir un discurso para una celebración en el instituto. Mientras que la madre la anima a ser ella misma y defender valores propios de la sociedad estadounidense como la independencia, el padre monta en cólera y rompe el discurso tras oírlo porque le parece irrespetuoso y subversivo, ya que los valores de humildad, obediencia y sumisión son fundamentales en la cultura dominicana. Sin embargo, tras el estallido de rabia de Carlos, Laura no se pliega al modelo de sumisión que se espera de ella, sino que se enfada con él y le hace ver la realidad: "This is America, Papi, America! You are not in a savage country anymore" (ibid.: 146).

El papel de la madre como mediadora entre el padre y las hijas se nos muestra en repetidas ocasiones, así como también su manera de apoyarlas en sus estudios, sus decisiones y, en general, en la búsqueda de su lugar dentro de la sociedad estadounidense. Por ejemplo, es Laura la que siempre asiste orgullosa a los recitales de Yolanda. Esta actitud, como ya hemos dicho, se corresponde con una mayor adaptación al nuevo contexto, porque la madre también trata de encontrar su sitio en el nuevo país, y con un mejor manejo del idioma que le permite interactuar fácilmente con el entorno. Como veremos más adelante, esta correspondencia entre dominio de la lengua, adaptación y diferentes actitudes respecto al nuevo contexto se hace patente también entre la madre y las hijas: Laura habla inglés mejor que su marido, pero peor que sus hijas, quienes, por lo tanto, también se nos muestran más adaptadas a la cultura estadounidense y más emancipadas como mujeres. De esta manera, se hace obvio que el lenguaje constituye un elemento poderoso y fundamental en la lucha por la independencia, ya que es el medio para traducirse a uno mismo.

\section{LENGUAJE, IDENTIDAD Y ADAPTACIÓN}

El lenguaje es la señal más clara de nuestra identidad nacional, dice Hanna (2005: 112), y es cierto, aunque cabría añadir que la forma en que lo utilizamos revela mucho más acerca de nosotros. Porque, si bien la lengua que hablamos nos hace miembros de una determinada comunidad, de una colectividad, el modo en que convertimos esa lengua en el vehículo de expresión de nuestra identidad individual se convierte en una manera de decir quiénes somos en relación con el mundo que nos rodea. Así, cada pequeño matiz, cada elección que hacemos como hablantes, tiene un significado más profundo, porque las lenguas no cumplen un simple objetivo pragmático de comunicación, sino que son el medio con el que transmitir quiénes somos, al tiempo que nos recuerdan que "no hay palabras neutras ni inocentes" (Vidal Claramonte, 2007: 48).

El lenguaje es, pues, un instrumento básico de comunicación, pero también de autodefinición, especialmente en el caso de individuos híbridos 
como los personajes de esta novela. Para las personas que, como los García, habitan e interaccionan en más de un contexto cultural, social y geográfico responder a una pregunta en apariencia tan simple como la que hace referencia al lugar del que uno es, al que se siente ligado por una relación de pertenencia, resulta una tarea complicada y desconcertante. De hecho, es este un tema que se repite a lo largo de la novela, ya que la familia, especialmente las hijas, experimentan a menudo lo que numerosos autores denominan a sense of displacement, un sentimiento de des-plazamiento, de no pertenencia a ningún sitio. Dado que el arraigo constituye un punto de referencia esencial en las primeras etapas de creación de la identidad, este uprooting provoca una sensación de desorientación y pérdida que puede paliarse de numerosas formas, desde la adhesión total y absoluta a una de las culturas en juego (y por lo tanto también a la lengua que la representa ${ }^{2}$ hasta la búsqueda y aceptación de la mesticidad.

Así, las elecciones lingüísticas que hacen estas personas son conscientes y deliberadas, y esto lo sabe bien Álvarez, quien pone en boca de sus personajes determinados términos $y$ expresiones en una $u$ otra lengua de forma intencionada, proporcionándoles una carga significativa que debe estar presente

2 Ariel Dorfman relata un ejemplo curioso de este extremo en su autobiografía Heading South, Looking North. Sus padres emigraron a Estados Unidos siendo él un niño, a los pocos días de llegar Dorfman enfermó y estuvo internado en un hospital, aislado de su lengua materna, durante tres semanas. El escritor cuenta que estando allí se dio cuenta de que el español no le servía para comunicarse con los médicos y las enfermeras y, quizás debido a la angustia de la incomunicación, decidió rechazar su lengua materna y adoptar el inglés, por lo que, cuando le dieron el alta, sus padres se encontraron con que se negaba a hablar español. A partir de ese momento, Dorfman decidió de forma consciente construir su identidad mediante la adhesión total a la cultura estadounidense y la negación de sus raíces: «Home. That's were I was, where I had chosen to be: I was swinging low sweet chariot, come to carry me home, I was home, home on the range, I was in the land of the free and the home of the brave, this land was my land and it was made for you and me, but especially, I felt, it had been made for me» (Dorfman, 1998: 48).

Quizás este caso pueda parecer un poco extremo, pero no es tan raro si tenemos en cuenta que los niños y los adolescentes ( $\mathrm{y}$ a menudo también los adultos) quieren estar del lado de los «ganadores»y eso es precisamente lo que, en ese momento, representaba Estados Unidos para Dorfman. Al fin y al cabo, habían tenido que abandonar su país de origen, Argentina, debido a la persecución fascista y, recién llegado al lugar de destino, se había encontrado en una situación de desamparo en la que su idioma no le servía de nada. En el caso de Dorfman, como en muchos otros, la relación con la cultura de origen está marcada por un sentimiento de abandono. Su país les ha fallado, les ha obligado a marcharse a otro en el que no encajan porque les falla precisamente el instrumento fundamental: la lengua. La reacción tiene su lógica y, además, es un mecanismo adaptativo básico: dado que mi país y mi lengua me han abandonado, los rechazo y busco mi lugar en la nueva cultura que me ha acogido. Si además el nuevo país es Estados Unidos, un lugar cuyo inmenso poder asimilatorio ha sido sobradamente constatado por las oleadas de inmigrantes de todos los rincones del globo que han adoptado su cultura como propia, la reacción del Dorfman niño resulta incluso obvia: «Bereft of a past and a language that told me who I was, what else was I to do? I became an American» (ibid.: 50). 
en la traducción. Es evidente que cada miembro de la familia García evoluciona de manera diferente en este aspecto; al final de la historia, las hijas hablan inglés perfectamente, pero que no son capaces de expresarse de forma fluida en español; el padre sigue hablando un "broken English" (Álvarez, 1991: 25) y el inglés de la madre no evoluciona demasiado, sigue estando muy influenciado por la sintaxis española, como veremos a continuación. Obviamente, estos diferentes niveles de habla se corresponden con el nivel de adhesión a cada cultura de los personajes.

La hibridación lingüística de los García se hace patente en pequeños detalles a lo largo de toda la novela, por ejemplo, en el uso de los vocativos cariñosos. La madre utiliza "Cuquita" para las cuatro hijas y la nieta; las hermanas siempre llaman "Papi" a Carlos y "Mami" a su madre, excepto cuando se enfadan con ella y quieren que se sienta mal, en esas ocasiones utilizan la forma inglesa "Mom", un cambio bastante revelador:

Her daughters never called her Mom except when they wanted her to feel how much she had failed them in this country. She was a good enough Mami, fussing and scolding and giving advice, but a terrible girlfriend parent, a real failure of a Mom (ibid:: 135-6).

Esta distinción en los vocativos se mantiene en las dos versiones en español que existen de la obra, prueba de que ambos traductores estaban concienciados con el juego lingüístico que plantea la autora y sus implicaciones. No obstante, en el siguiente epígrafe veremos cómo, frente a problemas de traducción más complejos, tomaron decisiones que no siempre hacen justicia al texto original.

\section{LA FORMA DE HABLAR DE LAURA Y SUS TRADUCCIONES}

Antes de mostrar con ejemplos la particularidad de la forma de hablar del personaje de Laura y de analizar si la carga ideológica que hemos visto que implica se mantiene en las traducciones, quisiera contextualizar y proporcionar información sobre las dos versiones existentes en español.

La primera la realizó Jordi Gubern en 1994 para la colección Tiempos Modernos de Ediciones B. Gubern hace patente desde el principio que va a tratar de respetar el bilingüismo y la hibridación lingüística del original al incluir en la primera página una nota del traductor en la que dice que indicará con cursiva todas las palabras que figuran en español en el texto origen. Sin embargo, estas buenas intenciones quedan maltrechas con su empleo del español peninsular para dar voz a unos personajes que son dominicanos, una decisión que levantó cierta polémica: 
Esta versión de otra obra bilingüe contrarresta el monolingüismo como estrategia traductora normativa con la utilización de la cursiva como forma de marcar los vocablos consignados en el original en castellano, con el mantenimiento de ciertas expresiones inglesas en el texto traducido y con la inclusión de información cultural en notas del traductor a pie de página. Con todo, ¿bastan estas estrategias para calificar la traducción de subversiva? ¿O primará más en la evaluación el hecho de que el traductor emplee sistemáticamente la variedad peninsular del español para la caracterización de los personajes de ascendencia dominicana, una decisión (o estrategia por defecto) en la que cabe ver a la antigua potencia colonial en funcionamiento? (Martín Ruano 2003: 250-1).

Efectivamente, Álvarez no se mostró precisamente satisfecha con esta traducción en la que, por ejemplo, se utiliza el pronombre "vosotros" para la segunda persona del plural cuando éste no se utiliza en ningún país de America Latina. Es cierto que Gubern trata a menudo de mantener el juego entre lenguas en la traducción y que ofrece algunas soluciones realmente buenas, pero su estrategia no es sistemática, puesto que opta por mantener ciertos elementos en inglés, mientras que, como veremos, elimina otros que a menudo son más importantes.

La segunda versión fue realizada por Mercedes Guhl y revisada por Ruth Herrera para Vintage en 2007, aunque en España la podemos encontrar en la editorial Punto de lectura. Al parecer, Álvarez quedó mucho más contenta con esta nueva versión, porque Guhl es conocedora de la voz caribeña y, por lo tanto, respeta los modismos y giros idiomáticos dominicanos sin que eso afecte a la comprensión general del texto para otros hispanohablantes. En efecto, en esta versión se utiliza un español más cercano a la variante dominicana tanto en los términos (carros, guagua), como en el uso del pronombre "ustedes" para la segunda persona del plural ("Ustedes cuatro se pierden por allá" [Álvarez, 2007: 18], "No traigan a sus maridos a casa para mi cumpleaños" [ibíd: 39]). En cambio, no se marca de ninguna manera la presencia de vocablos españoles en el texto inglés y resulta curioso comprobar que tampoco se sigue una estrategia sistemática para las referencias culturales y lingüísticas.

Veamos ahora varios ejemplos de cómo caracteriza la autora al personaje de Laura mediante su particular forma de hablar y de cómo trasladan Gubern y Guhl este rasgo. En el original, se recalca en varias ocasiones que Laura habla inglés, pero no de forma totalmente correcta (por ejemplo, Yolanda afirma que "[mixes] up some common phrase" [ibid:: 7]) y, como hemos visto, éste es un elemento importante porque marca la distancia con sus hijas. Es decir, las niñas hablan un inglés perfecto y, por lo tanto, su adaptación 
al contexto norteamericano es mayor que la de su madre. De la misma manera, las niñas no hablan un español fluido, mientras que su madre sí lo hace. Los errores que comete y las características de su manera de expresarse pueden dividirse en dos grupos: en uno incluiríamos aquellos detalles cuyas causas son las interferencias con el español y en el otro grupo aquellos que revelan problemas con la lengua inglesa.

Así, en el primer grupo encontramos un rasgo que aparece en más novelas de autoras hispanas: la sintaxis está muy pegada al español, de manera que, a pesar de que Laura habla inglés, en ocasiones su forma de construir las frases no es inglesa en absoluto: "that Yo has always loved poetry" (ibid:: 47), "Bad men is what they've had!" (ibid.: 52). Esto no se observa en ninguna de las dos traducciones, si bien es cierto que es difícil trasladar esta marca a nuestro idioma. Otra característica que podríamos incluir en este grupo es la presencia de palabras en castellano en el discurso inglés de la madre. Ya habíamos comentado que Gubern sí señala estos términos marcándolos en cursiva, en cambio, Guhl no lo hace y tampoco opta por una estrategia de compensación para equilibrar la pérdida.

En el segundo grupo encontramos problemas como la mala pronunciación o construcción de algunas palabras: "Remember that time we took the car to Bear Mountain, and we re-ah-lized that we had forgotten to pack an opener with our pick-a-nick?' (Her daughters kept correcting her, but she insisted this was how it should be said.)" (ibid.: 137). A pesar de que la incorrección en la escritura desaparece en la versión de Gubern, sí se mantiene la referencia al error de la madre: "¿Recuerdas aquella vez que fuimos en coche a Bear Mountain y descubrimos que habíamos olvidado meter un abrelatas en la cesta de la comida? - Pronunciaba a su manera muchas de las palabras inglesas, pero sus hijas sabían que corregirla era inútil” (Álvarez, 1994: 141). Ambos rasgos desaparecen por completo en la traducción de Guhl: "¿Recuerdas aquella vez que fuimos a la Montaña del Oso y allí nos dimos cuenta de que habíamos olvidado llevar un abrelatas y cuando quisimos comer no teníamos con qué abrir las latas?” (Álvarez, 2007: 169-70).

También incluiríamos en este segundo grupo dos escenas de la historia en las que se hace patente de forma muy sutil que Laura es capaz de hablar inglés, pero que no es su primera lengua, que no lo domina lo suficiente como para, por ejemplo, recitar algo de memoria con facilidad. La primera escena ocurre cuando Laura va a un recital de poesía a escuchar a Yolanda y trata de recitar unos versos de un poema de Poe que no consigue recordar bien:

Many many years ago, something... something,

In a... something by the sea...

A princess there lived whom you may remember

By the name of Annabel Lee... (Álvarez 1991: 50). 
Gubern opta por traducirlo al español, y no sería una decisión desacertada si no fuera porque se pierde la referencia lingüística, ya que el hecho de no recordar bien el poema probablemente se debe en parte a que está en inglés. Guhl, en cambio, mantiene el texto igual que aparece en el original y lo traduce en una nota a pie de página en la que, además, explica a qué poema se están refiriendo.

La segunda escena tiene lugar cuando Yolanda está tratando de escribir un discurso para una fiesta en la escuela y la madre le pone como ejemplo el famoso discurso que Lincoln pronunció en Gettysburg. Al intentar citar la primera frase, Laura se equivoca y, en lugar de Four score and seven years ago, dice "Four score and once upon a time ago" (ibid.: 142). Gubern elimina por completo la referencia y simplemente dice: "le salió uno de los discursos más bellos de la historia" (Álvarez, 1994: 145), mientras que Guhl mantiene la frase original y explica la situación en una nota a pie de página que reproduzco a continuación:

Como sucede con tantas otras cosas que cita, Laura García no acierta con las primeras palabras del discurso que Abraham Lincoln pronunció en Gettysburg, donde se libró una de las grandes batallas de la Guerra de Secesión, que dicen: Four score and seven years ago [Hace ochenta y siete años], refiriéndose al momento de la independencia de Estados Unidos, y las mezcla con el comienzo tradicional de los cuentso de hadas Once upon a time [érase una vez] para terminar con algo que en español podría traducirse como "Hace ochenta años, había una vez...", y que no tendría mucho sentido en boca de Lincoln” (Álvarez 2007: 175).

Por último, el rasgo que más se repite a lo largo de la novela y que sin duda más caracteriza la manera de hablar de Laura es su mal uso de los refranes y las frases hechas: "her English was a mishmash of mixed-up idioms and sayings" (Álvarez, 1991: 135). En ocasiones los traduce literalmente del español, aunque la mayoría de las veces intenta emplear una expresión inglesa, pero la utiliza mal provocando un efecto cómico y mostrando, una vez más, que su manejo del inglés dista mucho de ser perfecto: "While she makes herself understood, her language also identifies her as an outsider, despite her attempts to assimilate" (Johnson, 2005: 70). Estos errores, como bien dice Johnson, la identifican como inmigrante y muestran su desplazamiento dentro de la cultura estadounidense.

Veamos un ejemplo de traducción literal al inglés de un refrán dominicano: “"Maybe not. Maybe, just maybe, there’s something they've missed that's important. With patience and calm, even a burro can climb a palm.' This last was one of her many Dominican sayings she had imported into her 
scrambled English" (Álvarez, 1991: 138). El dicho original dice "con paciencia y calma sube un burro a una palma" y así es como opta por traducirlo Guhl: “-Quizá no. Tal vez hayan pasado por alto algo importante. Con paciencia y calma, hasta un burro se sube a una palma. -Ése era uno de los muchos refranes dominicanos que había logrado importar a su revoloteado inglés" (Álvarez, 2007: 171). Curiosamente, Gubern no utiliza la expresión dominicana, sino que opta por proponer su propia versión ("Con paciencia y calma, incluso un burro puede trepar a una palmera" [Álvarez, 1994: 142]), lo que, en mi opinión, no es una decisión muy acertada, ya que para los lectores españoles, el refrán original también tiene una carga exótica que se podría haber aprovechado. Además, al perder la rima, se pierde también gran parte del efecto estilístico.

Sin embargo, las situaciones más difíciles de trasladar son aquellas en las que el efecto se produce por un mal uso de un dicho inglés. Analicemos varios para tratar de identificar las estrategias de los traductores respecto a este problema de traducción. Por ejemplo, en un momento dado Laura dice que está "green behind the ears" (Álvarez, 1991: 135) en lugar de wet behind the ears, las versiones españolas utilizan dos expresiones castellanas "tenía 'el oído verde" (Álvarez, 1994: 139) y "aún estaba en pañales" (Álvarez, 2007: 167). Más tarde afirma "when in Rome, do unto the Romans" (Álvarez, 1991: 135) y ambos traductores optan por el conocido refrán español "donde fueres, haz lo que vieres". Es decir, para el lector castellano, Laura no comete ningún error y ésta es una pérdida importante, porque, sumada a otras muchas que hemos comentado anteriormente, cambia por completo la imagen del personaje.

Apenas unas líneas después, afirma: "sticks and stones don't break bones" (id.), confundiendo el comienzo del dicho inglés Sticks and stones may break my bones, but words will never burt me. Guhl lo adapta utilizando y cambiando un dicho español: "A pedradas necias, oídos sordos" (Álvarez, 2007: 167). Por el contrario, Gubern mantiene la expresión en inglés y escribe una nota a pie de página explicando lo que ocurre:

"Palos y piedras no rompen huesos." Laura cita erróneamente un proverbio inglés que dice precisamente todo lo contrario: "Palos y piedras romperán mis huesos, pero los insultos (o 'las palabras', según el caso) no me herirán" (Álvarez, 1994: 139).

El último ejemplo aparece en la página 142, cuando Laura afirma: "necessity is the daughter of invention" (Álvarez, 1991) y ambos traductores lo traducen de forma literal como "la necesidad es la hija de la invención", es raro que ninguno haya optado por cambiar la versión castellana del refrán: "la necesidad/prudencia/experiencia es la madre de la ciencia". 
Como vemos, la variedad de soluciones que ofrecen Gubern y Guhl es amplia, sin embargo, llama la atención el hecho de que ninguno de los dos sea sistemático en la forma de tratar este problema. Unas veces adaptan los refranes al español, otras optan por soluciones más extranjerizantes y en ocasiones simplemente los traducen de forma de literal de manera que la situación pierde toda la gracia.

Si examináramos por separado cada uno de los pequeños detalles lingüísticos que Álvarez utiliza para caracterizar la forma de hablar de Laura, podríamos caer en el error de pensar que no es tan importante mantenerlo en la traducción. Sin embargo, analizando la obra en conjunto, observamos que la autora construye el personaje de la madre fundamentalmente a través de su lenguaje, instrumento que también utiliza para establecer diferentes niveles de adaptación y relación entre los protagonistas de la historia. Por lo tanto, el error de los traductores de no emplear una estrategia sistemática y homogénea para tratar esta cuestión provoca que la identidad de Laura aparezca desdibujada y borrosa en ambas versiones.

\section{CONCLUSIONES}

En How the Garcia Girls Lost Their Accents, Julia Álvarez nos presenta a las hermanas como a las cuatro protagonistas de la obra; sin embargo, los personajes secundarios del padre y de la madre son fundamentales para la historia, no sólo por su evidente papel en el desarrollo de los acontecimientos, sino también por su función como contrapunto a la identidad y personalidad de Carla, Sandra, Yolanda y Sofía. Para comprender la forma en que se enfrentan a las diversas situaciones, así como la evolución de sus caracteres y sus vidas, necesitamos las figuras de Carlos y Laura. Si bien tanto los personajes de las hijas, variados pero con similares características de rebelión y adaptación a la cultura estadounidense, como el de Carlos, conservador y estático a lo largo de la novela, quedan definidos de forma muy clara hasta el punto en que podríamos dibujar una línea en la que el padre estuviera en un extremo y Sofía (la más rebelde de las hermanas) en el otro, la madre, como hemos visto, es un personaje más ambivalente. Álvarez nos muestra su identidad con dos técnicas: de un lado, por oposición con los extremos que representan sus hijas y su marido, Laura no es tan cerrada ni reaccionaria como Carlos, pero tampoco es tan abierta y liberal como sus hijas, sino que funciona como mediadora, como traductora, en la difícil relación entre ambas partes. Del otro, mediante su particular forma de utilizar el lenguaje que muestra su malogrado intento de adaptación a la sociedad estadounidense ya que "as a primary marker of national identity, language and her dificultties with it become an obstacle to 
assimilation, demonstrating linguistically her dis-integration in American culture" (Johnson, 2005: 70).

Es obvio, por lo tanto, que la manera de hablar de Laura tiene una función de cohesión y definición en la novela que también debería estar presente en la traducción. Sin embargo, las dos versiones que existen de la novela en nuestro idioma no tratan esta cuestión de una forma coherente, por lo que gran parte de la información sutilmente proporcionada en el original mediante los errores, las interferencias y los olvidos de Laura desaparece en los textos españoles. Es fundamental, como traductores, ser conscientes de que, a menudo, los pequeños detalles que parecen no tener importancia a primera vista forman parte de un conjunto de rasgos que, a nivel macrotextual, sientan los pilares sobre los que se desarrollará el complejo entramado de una historia.

\section{BIBLIOGRAFÍA}

Álvarez, Julia (1991): How the Garcia Girls Lost Their Acents. Nueva York, Plume.

- (1994): De cómo las chicas García perdieron su acento. Barcelona, Ediciones B. Traducción de Jordi Gubern.

- (2007): De cómo las muchachas García perdieron el acento. Madrid, Punto de lectura. Traducción de Mercedes Guhl.

Cronin, Michael (2006): Translation and Identity. Londres/Nueva York, Routledge.

Dorfman, Ariel (1998): Heading South, Looking North: A Bilingual Journey. Londres, Sceptre.

ESQUivel, Laura (2007 [2005]): Malinche. Madrid, Punto de lectura.

HANNA, Sameh F. (2005): "Othello in Egypt. Translation and the (Un)making of National Identity" en Juliane House, $M^{\mathrm{a}}$ Rosario Martín Ruano y Nicole Baumgarten (eds.): Translation and the Construction of Identity. Seul, IATIS, 109-128.

Johnson, Kelli Lyon (2005): Julia Alvarez: writing a new place on the map. Albuquerque, University of New Mexico Press.

MARTÍN RuANO, Ma Rosario (2003): "Una (re)visión de la mirada sobre lo otro: el discurso crítico de los estudios de traducción y sus límites" en $\mathrm{M}^{\mathrm{a}}$ Carmen África Vidal Claramonte, Juan Crespo y $\mathrm{M}^{\mathrm{a}}$ Rosario Martín Ruano (eds.): Panorama actual de la investigación en traducción e interpretación. Granada, Atrio, 241-255.

Vidal Claramonte, Ma Carmen África (2007): Traducir entre culturas: diferencias, poderes, identidades. Berna, Peter Lang. 